\title{
An unforgettable debate between descriptive and experimental biology in the 1930s in China
}

\author{
Ming $\mathrm{Li}^{1}$, Zonggang $\mathrm{Hu}^{2}$, Le Kang ${ }^{1}$ \\ ${ }^{1}$ Beijing Institutes of Life Science, Chinese Academy of Sciences, Beijing 100101, China \\ 2 Lushan Botanical Garden, Jiangxi Province and Chinese Academy of Sciences, Jiujiang 332900, China
}

At the early 20th century, biological research was advancing with big steps, as new disciplines like genetics, development and metabolism had achieved tremendous success thanks to new experimental approaches. Meanwhile, China's biological research, which did not exist in many senses before the 20th century, was also going through a prosperous period between the 1920 s and 1937.

With the efforts of numerous overseas-trained young scientists, quite a few Chinese universities set up biology departments, the first one being the Department of Biology at the Southeast University, Nanjing. In 1922, the Science Society of China established a major biological research institute in Nanjing - the Biological Laboratory of the Science Society of China (BLSSC). The rapid development of Chinese biology attracted attention from all sectors of the community, thus receiving major financial support from foundations such as the China Foundation for the Promotion of Education and Culture (the China Foundation in short), an organization set up in 1924 to manage the Boxer Indemnity Fund. By the 1930s, there have been four biological institutes in China, mainly working on taxonomy and morphology. Together with the ever-expanding specimen collections in universities, taxonomy was reportedly the most advanced discipline of Chinese biological research then.

When taxonomy was making major progress in China, some foreign-educated biologists, especially the ones working on experimental biology, started to sense the differences between Chinese biological research and that of Western countries. The most outspoken person was Dr. Jingxi Wang (Fig. 1), an American-trained psychologist who was first recruited back by Sun Yat-sen University, Guangzhou, and later worked at Peking University as a professor of psychology.

Starting in 1932, Jingxi Wang published a series of articles in Independent Review, a journal advocating freedom of speech and providing an open platform for scholars to debate.

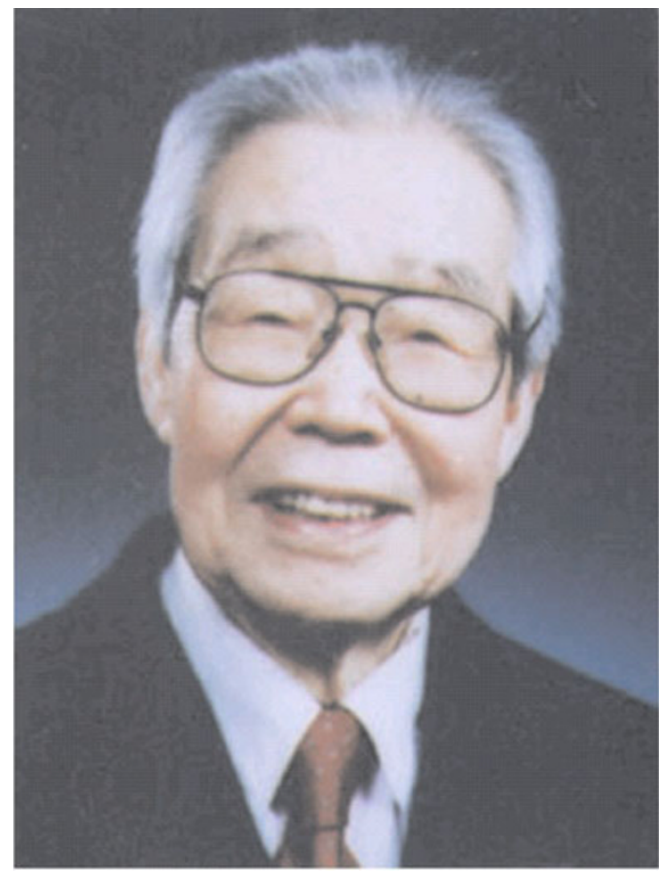

Figure 1. Dr. Jingxi Wang

Wang first pointed out that the trend of development of biological research in the Western countries was replacing descriptive/classical biology (taxonomy, morphology, fauna and flora, etc.) with experimental biology (genetics, physiology, etc.). Even though Chinese biology was merely at the starting point, it was important that it should be steered toward the right direction (i.e. experimental biology). Furthermore, he wrote, experiment-based causal explanations were proven more valuable in both theoretical and practical aspects, compared with taxonomy, which mainly described and named organism species. He also explained why experimental 
biologists could not get as much funding as taxonomists in China-the results of experimental biology (numbers, formulas, and figures) seemed less interesting to laymen than those of taxonomy (mainly based on animal and plant specimens), thus were underappreciated by the community. However, he added, experimental biology in China had a good start as many talented overseas Chinese scientists in the area had been recruited back to China, and it was in urgent need of financial support to be pushed forward.

Unsurprisingly, Wang's articles caused controversial discussion among Chinese biologists. Dr. Xiansu Hu (Fig. 2), a Chinese botanist who was the co-founder of the BLSSC, was the first one to refute. Hu's first argument was that even though the experimental biology was the dominant mode of research internationally, taxonomy was never falling behind in any countries. Instead of jumping with leaps, scientific development in China should take its course, like most Western countries. Especially for China, a country home to species diversity, credible biological research depended on accurate species identification and reliable scientific nominations. He also made comments about why taxonomy was getting more financial support: the development of a scientific discipline was mainly dependents on how much effort the scientists put into it, and it was reasonable that the community tended to support more successful disciplines. In fact, considering the vast biological resources in China, the funding for taxonomy was hardly sufficient to investigate local biological species.

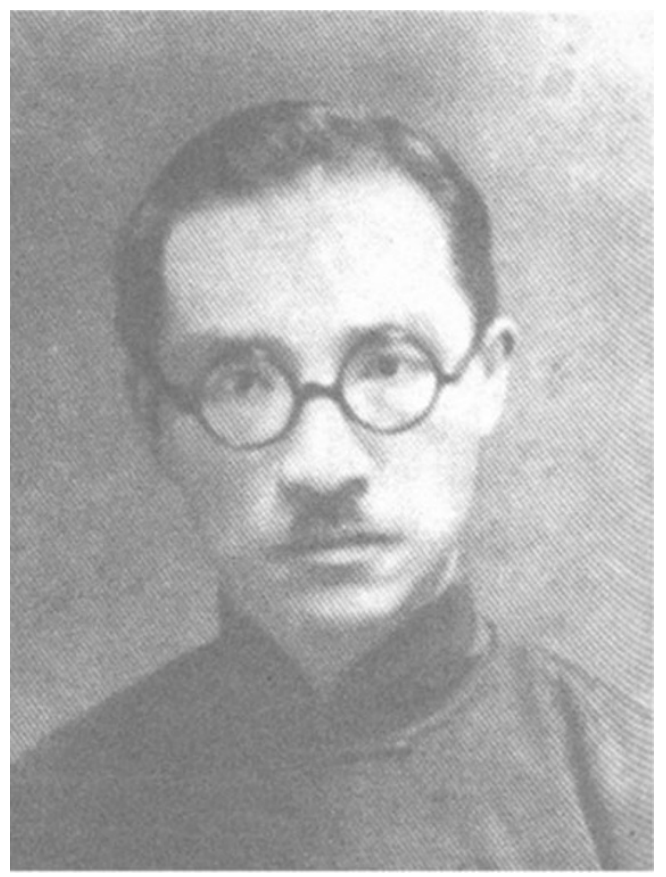

Figure 2. Dr. Xiansu Hu

The debate lasted four years, from 1932 to 1936 . Quite a few biologists got involved in the debate, including Zhenru
Wang and Zuoren Zhang. It also caused a debate among the benefactors and foreign advisors. The American entomologist J. G. Needham argued that most English-language botany and zoology textbooks used in Chinese universities were based on the natural environments of North America and Europe, not on that of China's own and that local species identification and investigation would provide a solid foundation for any further research. Meanwhile, there were other voices pointing out that the China Foundation was too singleminded in the way it chose one discipline over the others to support and that the scope of its beneficiary should expand from taxonomists to all biologists.

It was hard to say who won and who lost in the long debate. After all, the debate was never directed towards anyone involved personally; instead, both sides shared the same purpose-trying to promote the development of Chinese biological research. Unfortunately China was at a very unstable stage politically then and limited funding for scientific research was far from enough for ambitious scientists. It was not anyone's fault that one discipline was dominant over the others. It was the lack of coordination by the government's science policy that caused the imbalance among them before the World War II.

You may ask what happened after the debate. Well, Jingxi Wang never got the funding from the China Foundation and he eventually left China to permanently stay in the United States since 1940s. His peers such as geneticist Jiazhen Tan and plant physiologist Zongluo Luo stayed in China despite the hard struggle they had to go through to survive, and finally both made major achievements in their own areas. And Xianshu $\mathrm{Hu}$ also started to recruit non-taxonomists like geneticist Zhen Chen to join his department at National Central University, Nanjing (formerly known as the Southeast University). However, the impact of the debate on the history of Chinese biology should be examined not in the short term but in the long run. After the World War II, a large number of overseas Chinese scientists of many different disciplines returned to China and through their training, more young scientists became experts in various areas eventually. After the founding of the People's Republic of China, the curriculums of most biology departments in China were designed in a more balanced way; and Chinese experimental biology made some progress in genetics, biochemistry, physiology, ecology, etc. Especially since the 1970s, Chinese biological research started to advance at full speed and have made remarkable achievements in all disciplines. We believe that our predecessors like Jingxi Wang and Xiansu Hu would approve and appreciate today's Chinese biology, no matter which side they were on during the debate.

\section{REFERENCES}

Hu, Z. (2005). Unforgettable Xiansu Hu. Wuhan, Changjiang Literature and Art Publishing House. (胡宗刚. (2005). 不该遗忘的 胡先骕. 武汉, 长江文艺出版社.) 
Qian, Y., and Wang, Y. (2004). Chinese Academic Canon in the 20th Century: Biology. Fuzhou, Fujian Education Press. (钱迎倩, 王亚 辉. (2004). 20世纪中国学术大典: 生物学. 福州, 福建教育出版社.)
Schineider, L. (2005). Biology and revolution in twentieth-century China. Maryland, Rowman \& Littlefield Publishers, Inc. 